\title{
Enhancing sulforaphane absorption and excretion in healthy men through the combined consumption of fresh broccoli sprouts and a glucoraphanin-rich powder
}

\author{
Jenna M. Cramer, Margarita Teran-Garcia and Elizabeth H. Jeffery* \\ Department of Food Science and Human Nutrition, University of Illinois at Urbana-Champaign, 905 South Goodwin \\ Avenue, Urbana, IL 61801, USA \\ (Submitted 3 May 2011 - Final revision received 23 June 2011 - Accepted 12 July 2011 - First published online 13 September 2011)
}

\section{Abstract}

Sulforaphane (SF) is a chemopreventive isothiocyanate (ITC) derived from glucoraphanin (GRP) hydrolysis by myrosinase, a thioglucoside present in broccoli. The ability of broccoli powders sold as supplements to provide dietary SF is often of concern as many supplements contain GRP, but lack myrosinase. In a previous study, biomarkers of SF bioavailability from a powder rich in GRP, but lacking myrosinase, were enhanced by co-consumption of a myrosinase-containing air-dried broccoli sprout powder. Here, we studied the absorption of SF from the GRP-rich powder used in the previous study, but in combination with fresh broccoli sprouts, which are commercially available and more applicable to the human diet than air-dried sprout powder. A total of four participants each consumed four meals (separated by 1 week) consisting of dry cereal and yogurt with sprouts equivalent to $70 \mu \mathrm{mol}$ SF, GRP powder equivalent to $120 \mu \mathrm{mol}$ SF, both or neither. Metabolites of SF were analysed in blood and urine. The $24 \mathrm{~h}$ urinary SF- $N$-acetylcysteine recovery was 65,60 and $24 \%$ of the dose ingested from combination, broccoli sprout and GRP powder meals, respectively. In urine and plasma, ITC appearance was delayed following the GRP powder meal compared with the sprout and combination meals. Compared with the GRP powder or sprouts alone, combining broccoli sprouts with the GRP powder synergistically enhanced the early appearance of SF, offering insight into the combination of foods for improved health benefits of foods that reduce the risk for cancer.

Key words: Broccoli: Cancer: Glucoraphanin: Sulforaphane

Sulforaphane (SF), found in broccoli as its inactive precursor glucoraphanin (GRP), is considered to be responsible for the reduction of cancer risk that is associated with broccoli consumption. Upon crushing or chewing of fresh broccoli or broccoli sprouts, GRP is hydrolysed to SF by the plant thiohydrolase myrosinase. In instances of myrosinase inactivation, such as overcooking of broccoli, GRP can be hydrolysed to SF by microflora present in the lower gut ${ }^{(1,2)}$. However, GRP hydrolysis by microflora of the lower gut is far less efficient than hydrolysis by endogenous broccoli myrosinase ${ }^{(3-6)}$.

SF protects against the incidence and progression of cancer via several mechanisms including inhibiting phase I cytochrome P450 enzymes, inducing cell-cycle arrest and apoptosis, reducing inflammation, and perhaps most wellcharacterised, modulating the nuclear factor-erythroid-2related factor $2 /$ Kelch-like ECH-associated protein 1 pathway ${ }^{(7)}$. In the body, SF is metabolised by the mercapturic acid pathway and excreted in the urine, mostly as SF- $N$-acetylcysteine $(\mathrm{SF}-\mathrm{NAC})^{(8,9)}$. The fate of non-hydrolysed GRP is less well understood. A recent study has reported that low amounts of intact GRP were recovered in the urine of human subjects after consumption of a GRP-rich beverage, but not after consumption of an SF-rich beverage ${ }^{(9)}$. Another study has reported that low amounts of intact GRP were recovered in the urine, but not in the faeces of rats that were fed purified GRP ${ }^{(10)}$.

Several small clinical studies have examined the absorption and excretion of SF in human subjects. When urinary excretion of isothiocyanates (ITC) was measured following ingestion of fully cooked or fresh/lightly cooked broccoli, urinary ITC metabolites were approximately three times greater from fresh/lightly cooked broccoli than from fully cooked broccoli where myrosinase had been heat-inactivated ${ }^{(3,4)}$. Similar studies have evaluated the appearance of total ITC in the urine following ingestion of broccoli sprouts that had been either completely hydrolysed to ITC using exogenous myrosinase or contained only GRP where the myrosinase had been destroyed by boiling ${ }^{(5,9)}$. It has been found that ITC excretion was much greater after consumption of the preformed ITC compared

Abbreviations: GRP, glucoraphanin; ITC, isothiocyanate; SF, sulforaphane; SF-NAC, sulforaphane- $N$-acetylcysteine.

*Corresponding author: Professor E. H. Jeffery, fax +1 217265 0925, email ejeffery@illinois.edu 
with the GRP preparations ${ }^{(5,9)}$. The results of these studies suggest that the conversion of GRP to SF and subsequent ITC bioavailability is dependent on active myrosinase.

In a previous study, a commercially available GRP powder devoid of myrosinase, typical of many dietary GRP supplements on the market, was examined for its potential to deliver bioactive SF to human subjects alone or in combination with the air-dried broccoli sprout powder, which served as an exogenous food source of myrosinase ${ }^{(6)}$. The results showed that the combination improved the absorption of SF, and thus opened the door to the potential for enhanced cancer risk reduction not only from GRP supplements, but also from specifically designed foods or food combinations ${ }^{(6)}$.

Due to commercial availability and consumer preferences, intact fresh broccoli sprouts are more likely to be ingested by humans than the air-dried broccoli sprout powder used in our previous study. However, fresh broccoli sprouts may present additional variables such as matrix effects or product variability that were not present when examining the airdried broccoli sprout powder. Therefore, the present study examined the same commercially available GRP powder used in our previous study, but here, intact fresh broccoli sprouts were used as the exogenous food source of myrosinase. The present study also expanded the number of blood samples collected to better capture the differences in SF appearance in plasma from the test meals. The study sought to determine whether the fresh broccoli sprouts would enhance GRP conversion and ITC absorption from the GRP powder.

\section{Methods}

Fresh broccoli sprouts were donated by Tiny Greens Organic Farm (Urbana, IL, USA). Broccoli powder was a gift from Caudill Seed, Inc. (Louisville, KY, USA).

\section{Human subject study population}

A total of four healthy men, aged $18-30$ years, were recruited by fliers at the University of Illinois at Urbana-Champaign. Before participating in the study, each subject completed baseline questionnaires regarding dietary supplement, tobacco and other drug use. The present study was conducted according to the guidelines laid down in the Declaration of Helsinki and all procedures involving human subjects were approved by the University of Illinois Institutional Review Board. Written informed consent was obtained from all subjects. The study took place between 31 January and 25 February 2009.

\section{Study design, meal administration and sample collection}

Subjects were randomly assigned to a four-by-four cross-over design. They were given a list of foods known to contain glucosinolates and asked to avoid these foods for $3 \mathrm{~d}$ before and throughout the entire duration of the study. Subjects were also requested to avoid the use of dietary supplements and to limit alcohol consumption to no more than two alcoholic beverages per d during the study. Experimental meals were given each Tuesday for 4 weeks resulting in a 6 $\mathrm{d}$ washout period between meals; the half-life for SF has been reported as approximately $2 \mathrm{~h}^{(11,12)}$. On the morning of each trial, subjects were instructed to ingest a meal according to the cross-over design. In the case of sprout-containing meals, subjects were also instructed to chew the sprouts thoroughly. Meals included 5-d-old intact fresh broccoli sprouts of the calabrese variety (approximately $42 \mathrm{~g}$ ) or GRP powder $(2 \mathrm{~g})$ in an amount that produced 70 or $120 \mu \mathrm{mol}$ $\mathrm{SF}$, respectively, determined by bench hydrolysis. The combination meal contained both intact fresh broccoli sprouts (approximately $42 \mathrm{~g}$ ) and GRP powder (2g). The GRP powder was a proprietary dry, defatted broccoli seed powder preparation that did not contain myrosinase. Experimental meals were accompanied by one cup (53g) of dry cereal (Go Lean Crunch; Kashi Company, La Jolla, CA, USA) and half cup (113.5 g) of French vanilla fat-free yogurt (Stonyfield Farm, Londonderry, NH, USA) to serve as a vehicle and control meal. Thus, the control meal included cereal and yogurt only. Blood $(8 \mathrm{ml})$ was drawn into EDTA vacutainer tubes (Becton, Dickinson \& Company, Franklin Lakes, NJ, USA) immediately before ingestion of each meal $(0 \mathrm{~h})$, and at $0.5,1 \cdot 0,1 \cdot 5,3.0$ and $24 \mathrm{~h}$ following the meal. Plasma was immediately prepared by centrifugation and stored at $-80^{\circ} \mathrm{C}$ until analysed. Urine samples were collected at baseline (0 h), 0-6, 6-12 and 12-24h after meal consumption. All urine voided during these time intervals was collected. The volumes were recorded and used to calculate total $\mu \mathrm{mol}$ of SF-NAC excreted. Baseline urine samples were kept on ice and ascorbic acid (Fisher Scientific, Pittsburgh, PA, USA) was added to the urine samples at $1 \mathrm{~g} / 1$ urine no later than $1 \mathrm{~h}$ following collection. All other urine samples were collected into $500 \mathrm{ml}$ bottles containing $0.5 \mathrm{~g}$ ascorbic acid. Subjects were instructed to store urine samples in a provided cooler and to return them the following morning when the $24 \mathrm{~h}$ blood samples were collected. Urine samples were then stored at $-80^{\circ} \mathrm{C}$ until analysed.

\section{Sulforaphane analysis}

In triplicate, GRP powder $(50 \mathrm{mg}$ ) was added to $1.6 \mathrm{ml}$ distilled water containing $0.8 \mathrm{U}$ white mustard myrosinase (Sigma Chemical, St Louis, MO, USA), vortexed and left to hydrolyse in the dark for $24 \mathrm{~h}$. (One unit of myrosinase produces 1.0 $\mu \mathrm{mol}$ glucose $/ \mathrm{min}$ from sinigrin at $\mathrm{pH} 6.0$ and $25^{\circ} \mathrm{C}$.) The mixture was then centrifuged for $5 \mathrm{~min}$ at $14000 \mathrm{~g}$ and filtered through a $0.45 \mu \mathrm{m}$ nylon filter. The supernatant was diluted 5-fold with distilled $\mathrm{H}_{2} \mathrm{O}$ and an internal standard of benzyl ITC (Sigma Chemical) was added. The analysis of the GRP powder was also conducted in the absence of added myrosinase to confirm the necessity of myrosinase in the conversion of GRP to SF. Fresh broccoli sprouts were obtained the day before each trial meal and analysed for SF production upon hydrolysis using a modification of a previously reported method $^{(13)}$. In triplicate, $0 \cdot 25 \mathrm{~g}$ fresh broccoli sprouts were heated at $90^{\circ} \mathrm{C}$ for $5 \mathrm{~min}$ in a glass vial containing $2 \mathrm{ml}$ distilled water. Following heating, samples were cooled on ice, 
homogenised and $0.5 \mathrm{U}$ white mustard myrosinase were added. Samples were vortexed and left to hydrolyse at room temperature for $2 \mathrm{~h}$. The samples were then centrifuged for $5 \mathrm{~min}$ at $14000 \mathrm{~g}$. The supernatant was filtered through a $0.45 \mu \mathrm{m}$ nylon filter and diluted 4-fold with distilled water. An internal standard of benzyl ITC was added. ITC were extracted into dichloromethane for analysis by GC, as described previously ${ }^{(6)}$.

\section{Plasma total isothiocyanate analysis}

Blood samples were collected in EDTA-coated tubes and centrifuged at $1000 \boldsymbol{g}$ for $30 \mathrm{~min}$. Plasma was collected and analysed as described previously ${ }^{(6)}$. This method provides a single total measurement for SF, other ITC and metabolites ${ }^{(14,15)}$.

\section{Urinary sulforaphane- $\mathrm{N}$-acetylcysteine analysis}

Urine samples were analysed as described previously ${ }^{(6)}$. Briefly, the filtered urine $(50 \mu \mathrm{l})$ was analysed by HPLC using a Hypersil C18 ODS column $(10 \mu \mathrm{m}, 250 \times 4.6 \mathrm{~mm}$; Phenomenex, Torrance, CA, USA) and detected at $254 \mathrm{~nm}$ using a Waters HPLC system. A gradient solvent system with a flow rate of $1 \mathrm{ml} / \mathrm{min}$ consisted of a starting solvent system of $5 \%$ aqueous acetonitrile (Fisher Scientific) and 95\% water. Acetonitrile was increased linearly to $20 \%$ over $3 \mathrm{~min}$, held $4 \mathrm{~min}$, then increased to $100 \%$ over $2 \mathrm{~min}$ and held $13 \mathrm{~min}$ to rinse the column. Both solvents contained $1.0 \%$ acetic acid (Fisher Scientific). A standard was generated in control urine using SF-NAC synthesised as described previously ${ }^{(16)}$

\section{Statistical analysis}

Data were evaluated using the GLIMMIX procedure of SAS Statistical software (version 9.1; SAS Institute, Cary, NC, USA). Levels of SF metabolites in urine and blood were tested for interactions of treatment and time. Differences were separated using the SLICEDIFF option. Values were considered different at $P<0.05$.

\section{Results}

Sulforaphane content of hydrolysed broccoli sprouts and glucoraphanin powder

Upon incubation in water at room temperature for $24 \mathrm{~h}$ with the addition of $0.8 \mathrm{U}$ myrosinase, GRP powder produced
$61 \cdot 7(\operatorname{SE} 2 \cdot 1$ ) $\mu \mathrm{mol} \mathrm{SF} / \mathrm{g}$ powder. No SF was produced in the absence of added myrosinase. Fresh broccoli sprouts pro-

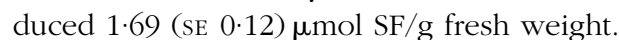

\section{Plasma total isothiocyanates}

Plasma ITC were elevated compared with baseline at $0.5 \mathrm{~h}$ in both the sprout and combination meals (Table 1 ). The combination meal reached peak plasma concentration (2.86 (SE $0.33) \mu \mathrm{mol} / \mathrm{l}) 1.5 \mathrm{~h}$ after ingestion. The sprout meal peaked at $3 \mathrm{~h}(1.53(\mathrm{se} 0.22) \mu \mathrm{mol} / \mathrm{l})$, but this value was not different from the value at $1.5 \mathrm{~h}(1.43(\mathrm{se} 0.21) \mu \mathrm{mol} / \mathrm{l})$. The GRP powder meal showed slightly elevated plasma concentration levels $3 \mathrm{~h}$ post-consumption (0.37 (sE 0.25) $\mu \mathrm{mol} / \mathrm{l}$ ). However, values following the GRP powder meal were not different from the control meal or baseline measurements at any of the time points measured. All values returned to baseline values by $24 \mathrm{~h}$.

\section{Sulforaphane- $\mathrm{N}$-acetylcysteine in the urine}

The amount of SF-NAC excreted in the urine over $24 \mathrm{~h}$ following ingestion of each meal is shown in Fig. 1. After ingestion of fresh broccoli sprouts in combination with GRP powder, individuals excreted a mean of 123.8 (SE 8.8) $\mu$ mol SF-NAC over $24 \mathrm{~h}$ post-ingestion, $65 \%$ of the dose ingested. After ingestion of broccoli sprouts alone, a mean of 42.0 ( $\mathrm{sE}$ 11.8) $\mu \mathrm{mol}$ SF-NAC was excreted, $60 \%$ of the ingested dose. However, after ingesting GRP powder alone, a mean of only $29 \cdot 2$ (SE 5.0) $\mu \mathrm{mol}$ SF-NAC was excreted, $24 \%$ of the dose ingested.

Urine collection was separated into discrete intervals for evaluation of SF-NAC excretion: urine was collected for the first $6 \mathrm{~h}$ after meal ingestion, from 6 to $12 \mathrm{~h}$ and from 12 to $24 \mathrm{~h}$ post-ingestion. Significant differences were observed between the dietary groups. Considerable levels of SF-NAC were excreted during the first $6 \mathrm{~h}$ from individuals who received the combination meal or the broccoli sprout meal (61 and $62 \%$ of the total SF-NAC that was excreted during the entire $24 \mathrm{~h}$ urine collection, respectively), but less than $22 \%$ of the total $24 \mathrm{~h}$ SF-NAC was excreted during this first $6 \mathrm{~h}$ period from those receiving the GRP powder meal alone. In contrast, less than $10 \%$ of the total $24 \mathrm{~h}$ SF-NAC recovered from the combination or sprout meal was excreted

Table 1. Total isothiocyanate (ITC) in plasma following test meal consumption (Mean values with their standard errors for four subjects per group)

\begin{tabular}{|c|c|c|c|c|c|c|c|c|c|c|c|c|}
\hline \multirow[b]{2}{*}{ Total ITC/I ( $\mu \mathrm{mol})$} & \multicolumn{2}{|c|}{$\mathrm{Oh}$} & \multicolumn{2}{|c|}{$0.5 \mathrm{~h}$} & \multicolumn{2}{|c|}{$1 \mathrm{~h}$} & \multicolumn{2}{|c|}{$1.5 \mathrm{~h}$} & \multicolumn{2}{|c|}{$3 \mathrm{~h}$} & \multicolumn{2}{|c|}{$24 \mathrm{~h}$} \\
\hline & Mean & SE & Mean & SE & Mean & SE & Mean & SE & Mean & SE & Mean & SE \\
\hline Control & $0.22^{a, A}$ & 0.09 & $0.18^{\mathrm{a}, \mathrm{b}, \mathrm{A}}$ & 0.11 & $0.24^{a, A}$ & 0.06 & $0 \cdot 16^{a, A}$ & 0.13 & $0.05^{\mathrm{a}, \mathrm{A}}$ & 0.03 & $0.05^{\mathrm{a}, \mathrm{A}}$ & 0.04 \\
\hline GRP powder & $0.09^{\mathrm{a}, \mathrm{A}}$ & 0.07 & $0.11^{\mathrm{a}, \mathrm{A}}$ & 0.07 & $0.07^{\mathrm{a}, \mathrm{A}}$ & 0.03 & $0 \cdot 12^{\mathrm{a}, \mathrm{A}}$ & 0.11 & $0.37^{\mathrm{a}, \mathrm{A}}$ & 0.25 & $0 \cdot 16^{\mathrm{a}, \mathrm{A}}$ & 0.10 \\
\hline Fresh sprout & $0.13^{\mathrm{a}, \mathrm{A}}$ & 0.12 & $0.46^{\mathrm{b}, \mathrm{A}}$ & 0.11 & $0.97^{\mathrm{b}, \mathrm{B}}$ & 0.15 & $1.43^{b, C}$ & 0.21 & $1.53^{\mathrm{b}, \mathrm{C}}$ & 0.22 & $0.19^{\mathrm{a}, \mathrm{A}}$ & 0.07 \\
\hline Combination & $0 \cdot 10^{\mathrm{a}, \mathrm{A}}$ & 0.07 & $1 \cdot 26^{\mathrm{c}, \mathrm{B}}$ & 0.22 & $2 \cdot 14^{\mathrm{c}, \mathrm{C}}$ & 0.15 & $2 \cdot 86^{\mathrm{c}, \mathrm{D}}$ & 0.33 & $2 \cdot 57^{\mathrm{C}, \mathrm{D}}$ & 0.38 & $0.30^{\mathrm{a}, \mathrm{A}}$ & 0.07 \\
\hline
\end{tabular}

GRP, glucoraphanin.

a,b,c Mean values within a column (between-meal values) with unlike superscript letters were significantly different $(P<0.05)$.

$A, B, C, D$ Mean values within a row (within-meal values) with unlike superscript letters were significantly different $(P<0.05)$. 


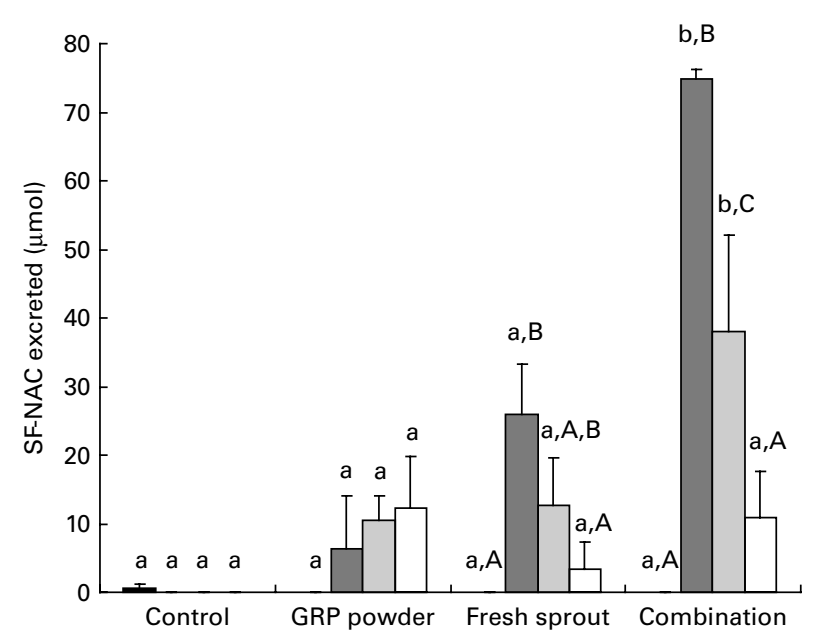

Fig. 1. Urinary sulforaphane- $N$-acetylcysteine (SF-NAC) excretion after consumption of four different meals: control; glucoraphanin (GRP) powder: fresh broccoli sprout; combination. Baseline $(\square), 0-6 \mathrm{~h}(\square), 6-12 \mathrm{~h}(\square)$ and $12-24 \mathrm{~h}(\square)$ urine collection post-consumption. Values are means, with their standard errors represented by vertical bars for four subjects per group. ${ }^{a, b}$ Mean values with unlike letters were significantly different between-meal $(P<0.05)$. ${ }^{\mathrm{A}, \mathrm{B}, \mathrm{C}}$ Mean values with unlike letters were significantly different within-meal $(P<0.05)$.

during the $12-24 \mathrm{~h}$ period, whereas $42 \%$ of the total $24 \mathrm{~h}$ SF-NAC recovered following the GRP powder meal was excreted during this later time period.

\section{Discussion}

The main findings of the present study were that combining fresh broccoli sprouts with the GRP powder (1) increased the appearance of SF metabolites in plasma and urine and (2) removed the delay of metabolite appearance observed after the GRP powder, shifting the absorption/elimination pattern to the one similar to that seen after the consumption of fresh broccoli sprouts alone. This is the first study to determine whether combining two commercially available broccoli products, one containing and the other lacking myrosinase, would enhance SF availability from GRP. The present study could be extrapolated to hypothesise that combining fresh broccoli sprouts with well-cooked broccoli, where myrosinase is inactive, would also enhance SF availability. Additionally, it could be hypothesised that other sources of myrosinase, such as mustard, horseradish, cabbage, Brussels sprouts and watercress, would also enhance the conversion of GRP to SF.

The present study measured urinary SF-NAC excretion and plasma total ITC levels. The measurement of SF metabolites after consumption of broccoli, broccoli sprouts and other broccoli-related preparations has been a useful tool for assessing human exposure to SF, a compound associated with a reduced risk of cancer ${ }^{(1,3-6,9,17,18)}$. SF metabolites in plasma reflect the amount of $\mathrm{SF}$ that tissues are being exposed to and are therefore important biomarkers of exposure to this cancer-preventive agent. SF metabolites in the urine reflect the absorption, metabolism and excretion of an ingested dose $^{(5)}$. The major metabolite of SF appearing in the urine,
SF-NAC, is often used as a marker of bioavailability, although it is not the only metabolite present in the urine ${ }^{(8,9)}$.

Only $24 \%$ of the GRP dose from the GRP powder was recovered as SF-NAC in the urine, making it a poor source of dietary SF compared with fresh broccoli sprouts. This value is comparable with the reported recovery following ingestion of well-cooked sprouts or well-cooked mature broccoli, both of which also lacked myrosinase ${ }^{(3,5,18)}$. Urinary values of SF-NAC after the GRP powder meal displayed a non-significant trend of increasing excretion over $24 \mathrm{~h}$, suggestive of delayed absorption. The delayed absorption and low SF recovery was probably due to the lack of myrosinase in the powder and the resulting hydrolysis of GRP by microflora after the transit of GRP to the lower gut ${ }^{(1,2,18,19)}$. Plasma total ITC was not altered in response to the GRP powder, but there was a slight non-significant elevation at $3 \mathrm{~h}$, also suggestive of delayed ITC absorption with low availability. It could be questioned whether the maximum plasma ITC level was further delayed rather than absent following ingestion of the GRP powder, and thus was not measured in the present study. Indeed, a slight, but non-significant peak $6 \mathrm{~h}$ postconsumption was observed in a study of well-cooked broccoli $^{(3)}$. However, urinary recovery of SF-NAC was low not only for the first $6 \mathrm{~h}$ of the present study, but for the entire $24 \mathrm{~h}$ period following consumption of the GRP powder meal, confirming the absence of any significant elevation in plasma ITC levels. The low levels of SF metabolites detected in plasma and urine after consumption of the GRP powder may indicate lower anti-cancer potential for this product and other similar dietary supplements. For instance, it has been reported that similar GRP products lacking myrosinase induced detoxification enzymes in the colon, but not in the liver of rats, whereas unheated broccoli florets with functional myrosinase induced activity in both the colon and the liver ${ }^{(20)}$.

Interestingly, data from the combination meal identified possible synergy among the fresh sprouts and GRP powder at early time points for SF and its metabolite appearance in plasma and urine. This indicates that GRP, not only from the broccoli sprouts, but also from the GRP powder, was hydrolysed by endogenous myrosinase from the broccoli sprouts. Additionally, excretion of SF-NAC following the combination meal was earlier than from the GRP powder alone and more similar to the excretion pattern following the consumption of broccoli sprouts alone, indicating that the fresh sprouts not only supported the hydrolysis of the GRP powder, but also caused it to occur earlier, resulting in earlier and more complete SF absorption. The trend for greater levels of SFNAC to be excreted early (during the first $12 \mathrm{~h}$ following meal consumption) from the combination and sprout meals is consistent with metabolism occurring in the upper gastrointestinal tract in the presence of dietary myrosinase. A similar trend was observed in plasma where in both sprout and combination meals, plasma ITC levels were elevated by $0.5 \mathrm{~h}$, and to a much higher level in the combination meal. Higher levels of SF metabolites in plasma and urine may indicate a greater reduction of cancer risk from consumption of this food combination. 
Recovery of preformed ITC or ground, air-dried broccoli sprouts has been reported to be between 75 and $90 \%$ of ingested doses ${ }^{(5,6,21)}$. This recovery decreased when a plant matrix was introduced, as is evidenced by several published papers, as well as the present paper where intact, but thoroughly chewed fresh sprouts resulted in a $60 \%$ recovery of the dose $\mathrm{e}^{(3,5,21)}$. Interestingly though, comparing an equimolar dose of SF from fresh sprouts (used here) with airdried sprouts ${ }^{(6)}$ when combined with the GRP powder, an improved $24 \mathrm{~h}$ urinary recovery (65 v. 50\% of the ingested dose, respectively) and an elevated peak plasma ITC level ( $C_{\max } 2 \cdot 9$ v. $2 \cdot 1 \mu \mathrm{mol}$ total ITC/1, respectively) was observed in the combination using fresh intact broccoli sprouts. Based on this evidence, we conclude that fresh broccoli sprouts aided the conversion of GRP to SF from the GRP powder to a greater extent than air-dried broccoli sprouts. More research with larger study populations is needed.

One limitation of the present study is its small sample size. However, most human studies focusing on the bioavailability of SF have used similar small population sizes ${ }^{(3,5,6,11)}$. The intent of the present study was to provide direction as a pilot study. Future large-scale work is needed.

\section{Conclusion}

GRP powder that lacked myrosinase was a poor dietary source of SF compared with broccoli sprouts. Fresh intact broccoli sprouts were able to synergistically enhance the hydrolysis of GRP from the GRP powder, perhaps more efficiently than ground, air-dried broccoli sprouts. Because efficacy is related to plasma levels, the elevation seen in plasma levels probably translates to a greater potential for cancer risk reduction. These findings provide important insights into the protective health benefit of broccoli products and preparations and can be used to develop foods with enhanced anti-cancer properties.

\section{Acknowledgements}

We thank Tiny Greens Organic Farm for supplying the fresh broccoli sprouts that were used in this study. We also thank John Jerrell for his help and expertise with HPLC analysis, as well as Heather Mangian and Melissa Sorrells for support in preparation for and during sample collection. J. M. C. was supported by a fellowship from Philip and Juanita Francis. This study received no specific grant from any funding agency in the public, commercial or not-for-profit sectors. The authors declare that they have no conflicts of interest. The authors' contributions were as follows: J. M. C. participated in the study design, completed Institutional Review Board application, carried out meal administration and sample collection, completed all analyses and drafted the manuscript. M. T.-G. carried out phlebotomy for blood sample collection. E. H. J. participated in the study design and oversight of Institutional Review Board application. All authors participated in the manuscript discussion as well as read and approved the final manuscript.

\section{References}

1. Shapiro TA, Fahey JW, Wade KL, et al. (1998) Human metabolism and excretion of cancer chemoprotective glucosinolates and isothiocyanates of cruciferous vegetables. Cancer Epidemiol Biomarkers Prev 7, 1091-1100.

2. Lai RH, Miller MJ \& Jeffery EH (2010) Glucoraphanin hydrolysis by microbiota in the rat cecum results in sulforaphane absorption. Food Funct 1, 161-166.

3. Conaway CC, Getahun SM, Liebes LL, et al. (2000) Disposition of glucosinolates and sulforaphane in humans after ingestion of steamed and fresh broccoli. Nutr Cancer 38, 168-178.

4. Rungapamestry V, Duncan AJ, Fuller Z, et al. (2007) Effect of meal composition and cooking duration on the fate of sulforaphane following consumption of broccoli by healthy human subjects. Br J Nutr 97, 644-652.

5. Shapiro TA, Fahey JW, Wade KL, et al. (2001) Chemoprotective glucosinolates and isothiocyanates of broccoli sprouts: metabolism and excretion in humans. Cancer Epidemiol Biomarkers Prev 10, 501-508.

6. Cramer JM \& Jeffery EH (2011) Sulforaphane absorption and excretion following ingestion of a semi-purified broccoli powder rich in glucoraphanin and broccoli sprouts in healthy men. Nutr Cancer 63, 196-201.

7. Herr I \& Buchler MW (2010) Dietary constituents of broccoli and other cruciferous vegetables: implications for prevention and therapy of cancer. Cancer Treat Rev 36, 377-383.

8. Al Janobi AA, Mithen RF, Gasper AV, et al. (2006) Quantitative measurement of sulforaphane, iberin and their mercapturic acid pathway metabolites in human plasma and urine using liquid chromatography-tandem electrospray ionisation mass spectrometry. J Chromatogr B Analyt Technol Biomed Life Sci 844, 223-234.

9. Egner PA, Chen JG, Wang JB, et al. (2011) Bioavailability of sulforaphane from two broccoli sprout beverages: results of a short-term, cross-over clinical trial in Qidong, China. Cancer Prev Res (Phila) 4, 384-395.

10. Bheemreddy RM \& Jeffery EH (2007) The metabolic fate of purified glucoraphanin in F344 rats. J Agric Food Chem 55 , 2861-2866.

11. Ye L, Dinkova-Kostova AT, Wade KL, et al. (2002) Quantitative determination of dithiocarbamates in human plasma, serum, erythrocytes and urine: pharmacokinetics of broccoli sprout isothiocyanates in humans. Clin Chim Acta 316, 43-53.

12. Vermeulen M, Klopping-Ketelaars IW, van den Berg R, et al. (2008) Bioavailability and kinetics of sulforaphane in humans after consumption of cooked versus raw broccoli. J Agric Food Chem 56, 10505-10509.

13. Matusheski NV, Juvik JA \& Jeffery EH (2004) Heating decreases epithiospecifier protein activity and increases sulforaphane formation in broccoli. Phytochemistry 65, 1273-1281.

14. Zhang Y, Cho CG, Posner GH, et al. (1992) Spectroscopic quantitation of organic isothiocyanates by cyclocondensation with vicinal dithiols. Anal Biochem 205, 100-107.

15. Zhang Y, Wade KL, Prestera T, et al. (1996) Quantitative determination of isothiocyanates, dithiocarbamates, carbon disulfide, and related thiocarbonyl compounds by cyclocondensation with 1,2-benzenedithiol. Anal Biochem 239, $160-167$.

16. Keck AS, Qiao Q \& Jeffery EH (2003) Food matrix effects on bioactivity of broccoli-derived sulforaphane in liver and colon of F344 rats. J Agric Food Chem 51, 3320-3327.

17. Hanlon N, Coldham N, Gielbert A, et al. (2009) Repeated intake of broccoli does not lead to higher plasma levels of sulforaphane in human volunteers. Cancer Lett 284, 15-20. 
18. Shapiro TA, Fahey JW, Dinkova-Kostova AT, et al. (2006) Safety, tolerance, and metabolism of broccoli sprout glucosinolates and isothiocyanates: a clinical phase I study. Nutr Cancer 55, 53-62.

19. Verhoeven DT, Verhagen H, Goldbohm RA, et al. (1997) A review of mechanisms underlying anticarcinogenicity by brassica vegetables. Chem Biol Interact $\mathbf{1 0 3}$, 79-129.
20. Zhu N, Soendergaard M, Jeffery EH, et al. (2010) The impact of loss of myrosinase on the bioactivity of broccoli products in F344 rats. J Agric Food Chem 58, $1558-1563$

21. Rouzaud G, Young SA \& Duncan AJ (2004) Hydrolysis of glucosinolates to isothiocyanates after ingestion of raw or microwaved cabbage by human volunteers. Cancer Epidemiol Biomarkers Prev 13, 125-131. 